\title{
Energy Efficiency of an Enhanced DCF Access Method using Bidirectional Communications for Infrastructure-based IEEE 802.11 WLANs
}

\author{
Raul Palacios ${ }^{\mathrm{a}}$, Fabrizio Granelli ${ }^{\mathrm{a}}$, Dzmitry Kliazovich ${ }^{\mathrm{b}}$, Luis Alonso ${ }^{\mathrm{c}}$ and Jesus Alonso-Zarate ${ }^{\mathrm{d}}$ \\ a \{palaciostrujillo, granelli\}@disi.unitn.it, University of Trento, Italy \\ bdzmitry.kliazovich@uni.lu, University of Luxembourg, Luxembourg \\ cluisg@tsc.upc.edu, Universitat Politècnica de Catalunya (UPC-EETAC), Barcelona, Spain \\ djesus.alonso@cttc.es, Centre Tecnològic de Telecomunicacions de Catalunya (CTTC), Barcelona, Spain
}

\begin{abstract}
The Distributed Coordination Function (DCF) is the fundamental access method defined in the IEEE 802.11 Standard for Wireless Local Area Networks (WLANs). With this standard, the Access Point (AP) and the mobile stations consume a significant amount of energy to contend for access to the shared wireless channel. In order to improve the energy efficiency of WLANs, we investigate in this paper a simple and backwards compatible mechanism, called Bi-Directional DCF (BD-DCF), that enables bidirectional communications between wireless stations with a single channel access invocation. The key idea is to let the AP or any mobile station transmit a data packet together with the acknowledgement upon the successful reception of a data packet. This approach reduces the communication overhead, the channel contention, and better balances uplink and downlink transmission opportunities. We evaluate the performance of the proposed BD-DCF by means of computer-based simulation considering different traffic loads, degrees of traffic symmetry, data packet sizes, and data rates. The results presented in this paper show that the BD-DCF protocol can improve the energy efficiency of DCF up to $50 \%$.
\end{abstract}

\section{INTRODUCTION}

Wireless Local Area Networks (WLANs) have been widely deployed in public and private areas due to their capability to provide both high data rates and user mobility in short range. A WLAN typically consists of an Access Point (AP) and multiple mobile stations, as shown in Fig. 1. The AP is connected to the infrastructure network to allow the mobile stations of the WLAN to access to a set of telecommunications services through the same shared wireless channel. Since many different types of mobile stations are currently equipped with WLAN interfaces, mobile users are increasingly adopting WLANs as an essential means of communications in daily social and business activities.

In hotspots, WLAN APs are heavily loaded and consume a significant amount of energy to offer continuous wireless connection to multiple mobile users [1]. In addition, mobile stations experience high energy consumption when they use the WLAN interface [2]. Mobile applications that require a huge amount of data, such as Skype or YouTube, contribute to the quick depletion of the batteries. Therefore, the WLAN

* This work is funded by the GREENET research project (PITN-GA-2010264759).

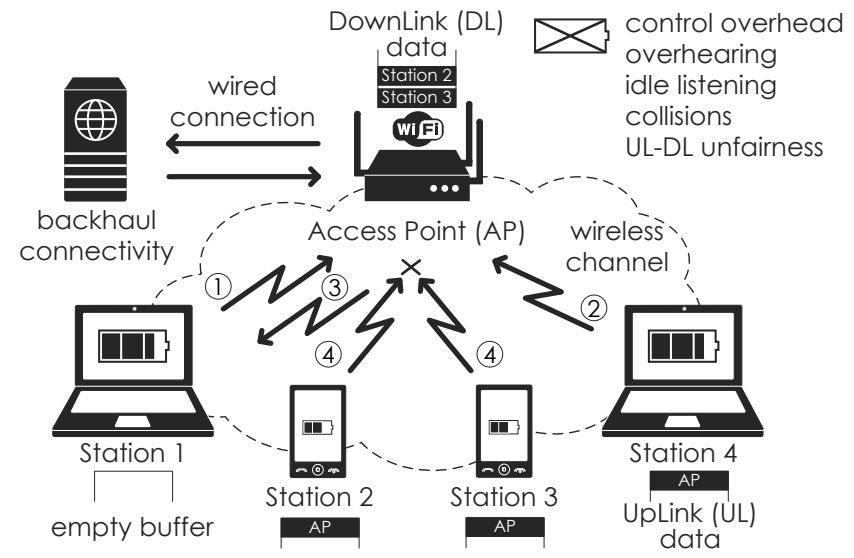

Fig. 1. An infrastructure-based WLAN where the AP and the mobile stations consume a significant amount of energy to compete for channel access.

technology should be optimized to satisfy the increasing traffic demand of mobile users and make wireless communications energy efficient at the same time.

The IEEE 802.11 Standard [3] defines the specification of the Medium Access Control (MAC) and physical (PHY) layers for WLANs. Two access methods are defined in this standard: the Distributed Coordination Function (DCF) and the Point Coordination Function (PCF). The DCF is mandatory for any compliant device and is widely implemented in commercial wireless cards. This access method is based on contention using Carrier Sense Multiple Access with Collision Avoidance (CSMA/CA) and a Binary Exponential Backoff (BEB) algorithm. It can be executed in both ad hoc and infrastructurebased networks. In its turn, the PCF is an optional pollingbased access method that can provide infrastructure-based networks with some degree of Quality of Service. This access method is rarely found in commercial WLAN interfaces.

The DCF defines a basic access mode and an optional CA mechanism implemented through a handshake of control packets called Request-To-Send (RTS) and Clear-To-Send (CTS). The basic scheme allows a source station to initiate the transmission of data without the need to establish connection with an intended destination station. Each transmission is positively acknowledged by the destination station with an 
ACK packet to notify the source station of successful reception of data. In addition, the RTS/CTS handshake can precede the transmission of actual data. This handshake aims to reduce the impact of packet collisions and can reduce the impact caused by the presence of hidden terminals.

When the DCF is executed, the AP and the mobile stations must sense the channel activity before transmitting data. Upon detecting a transmission, a backoff process of random duration is executed to minimize the probability of collisions with other transmitting stations. The overhead generated by the exchange of control packets, the channel sensing and backoff periods, and packet collisions are the main sources of energy consumption in DCF. For this reason, many solutions addressing these issues have been proposed in the literature [4].

In particular, the idea of introducing bidirectional communications in the rules of the DCF has been studied in several works [5]-[10] to increase capacity and throughput in IEEE 802.11-based wireless ad hoc networks. These approaches are based on the observation that the basic DCF only allows initiating unidirectional transmissions of data between pairs of wireless stations. With bidirectional transmissions, the destination station can exchange data with the source station through the basic and the RTS/CTS schemes. Therefore, it does not have to contend for the wireless channel, hence reducing the control overhead and overall contention in the network.

Different methods have been proposed to enable bidirectional communications in ad hoc communication mode. In [5], the destination station can use the ACK packet to reserve the wireless channel in the opposite direction. Then the source station replies with a CTS packet and the destination station initiates data transmission. The data exchange concludes with the ACK packet from the source station. The approach proposed in [6] allows the destination station to send a data packet after transmitting the ACK back to the source station. In [7], data can be attached to both the CTS and ACK packets. The work in [8] suggests that a Transmission Opportunity (TxOP) can be used to initiate multiple bidirectional transmissions. In a TxOP, the destination station may request a bidirectional transmission in the CTS packet. Then, the source station can grant the destination station a portion of the TXOP to transmit data in the reverse link. In [9] and [10], the receiver of an RTS packet becomes a temporary master using the PCF to transmit data and poll the mobile stations located in the coverage range.

However, we have observed that little attention has been paid to exploiting bidirectional communications in infrastructure-based WLANs. Up to our knowledge, the work in [11] can be considered as exception, wherein bidirectional transmissions are used to achieve fairness between the uplink and the downlink. In the long-term, the amount of channel access opportunities with the DCF are equal, in average, for all competing stations. However, if we take into account that the AP concentrates traffic from all the mobile stations, this long-run fair share of traffic opportunities yields some degree of unbalance between the downlink of the AP and the uplink of the mobile stations. In [11] the AP can transmit in the downlink by using the received data packet from any mobile station to balance uplink and downlink channel access opportunities, based on a fairness indicator.

The main contribution of this paper consists in defining and evaluating a simple and backwards-compatible mechanism, called Bi-Directional DCF (BD-DCF), that incorporates bidirectional communications in DCF to improve the energy efficiency of infrastructure-based WLANs. Unlike the approach presented in [11], the key idea of BD-DCF is to allow both the AP and the mobile stations to opportunistically exploit bidirectional transmissions while maintaining the balance between the uplink and the downlink. A preliminary definition and performance evaluation of the protocol was already presented in [12]. In this work, we extend [12] with a comprehensive performance evaluation and comparison of a BD-DCF-enabled network and a legacy DCF network. Computer-based simulation results are discussed in terms of the energy efficiency under different traffic conditions. The effects of the PHY data transmission rate, size of the data packet, and degree of traffic symmetry, are carefully analyzed to determine the gains that can be achieved in terms of energy efficiency by adaptively exploiting bidirectional communications in WLANs.

The remainder of the paper is structured as follows. Section II provides an overview of the DCF and the description of the BD-DCF. Section III introduces the performance evaluation and discusses the most relevant obtained results. In this section, the performance of BD-DCF is compared to that of the legacy DCF. Finally, conclusions are drawn in Section IV.

\section{Enhanced Channel ACCess}

The rules of the $\mathrm{BD}-\mathrm{DCF}$ protocol are essentially the same as those defined in the DCF. However, in BD-DCF, a destination station is allowed to transmit a data packet upon the reception of a data packet from a source station. Instead of simply transmitting an explicit ACK packet, the destination is able to transmit a data packet whose destination is the source station of the received packet. By enabling such bidirectional communication, it is possible to reduce channel contention and improve throughput, access delay, and energy efficiency.

\section{A. IEEE 802.11 DCF Overview}

The DCF specifies that a station with data to transmit has to first listen to the wireless channel. If the wireless channel is sensed idle for a DCF Inter Frame Space (DIFS), the station can transmit. Otherwise, it cannot transmit and has to update the Network Allocation Vector (NAV) according to the time indicated in the duration field of the MAC header of RTS, CTS, and data packets. The NAV accounts for the time that the wireless channel will be occupied. When the wireless channel is sensed idle again for a DIFS, the station waits for a random backoff time before transmitting the packet to avoid packet collisions. This period of time is determined by a backoff counter that is uniformly selected between 0 and $w-1$, where $w$ is the Contention Window $(\mathrm{CW})$. The $w$ value is set to the minimum $\mathrm{CW}$ size $\left(C W_{\min }\right)$ and doubles up to the maximum $\mathrm{CW}$ size $\left(C W_{\max }\right)$ for each failed retransmission attempt. The backoff counter is decremented by one whenever 
the wireless channel is sensed idle for a slot time. It is frozen whenever the wireless channel is sensed busy. The station initiates transmission when the backoff counter reaches zero. If another data packet has to be transmitted, the station will have to wait for a new random backoff time after a DIFS to avoid channel capture.

Fig. 2a illustrates an example of operation of the DCF with the energy profiles of all the stations during transmission (tx), reception (rx), and idle modes. The stations actively use the wireless interface to transmit and receive packets in the first two modes. In the idle mode they are powered on but no signal is present in the wireless interface. In this example, Station A and the AP exchange data by using the RTS/CTS access method. Prior to transmitting a new data packet, the AP randomly chooses a backoff counter equal to 8 and starts decrementing it after an idle DIFS. At $t_{0}$ Station A attempts to transmit a new data packet to the AP. After a DIFS, it gains channel access and initiates an RTS/CTS exchange before sending the data packet. At this time, the AP stops decrementing the backoff counter freezing it to 4 and replies to Station A with a CTS packet. After a Short IFS (SIFS), Station A sends the data packet and the AP acknowledges it with an ACK packet after a new SIFS. After a DIFS, the AP resumes decrementing its backoff counter up to 0 . Then it initiates a new RTS/CTS handshake to transmit a data packet to Station A. During the whole transmission, other contending mobile stations avoid attempting channel access until NAV expires.

This simple example shows that the data exchange between the AP and any mobile station is clearly inefficient by using the RTS/CTS access mechanism. The RTS and CTS packets are required for the transmission of a single data packet and an ACK packet is always necessary from the receiver station to acknowledge it. Silent periods are used to share the wireless channel among the stations and to allow them to switch their radio transceivers between the transmission and reception modes. When the channel activity increases, the stations have to wait for random backoff times to avoid packet collisions. As a result, their limited energy resources are spent not efficiently.

\section{B. The BD-DCF Protocol}

An example of operation of the BD-DCF is shown in Fig. 2b. This example follows the same description as in Fig. 2a to describe the DCF protocol. However, when the AP receives the RTS, it replies with a CTS that includes the value for the NAV associated to the duration of the entire bidirectional communication. This means that the value of the NAV accounts for the transmission time of the data packet in the forward direction, the data packet in the backward direction, the trailing ACK, and all the silent periods (SIFS). The other stations that received the CTS read the duration field and update their NAVs, thus making this mechanism fully compatible with the standard. After receiving the CTS, Station A initiates data transmission. Then, the AP replies with a data packet after successful reception. This newly received data packet is interpreted by Station A as an ACK to its own data packet, hence significantly reducing the amount of control

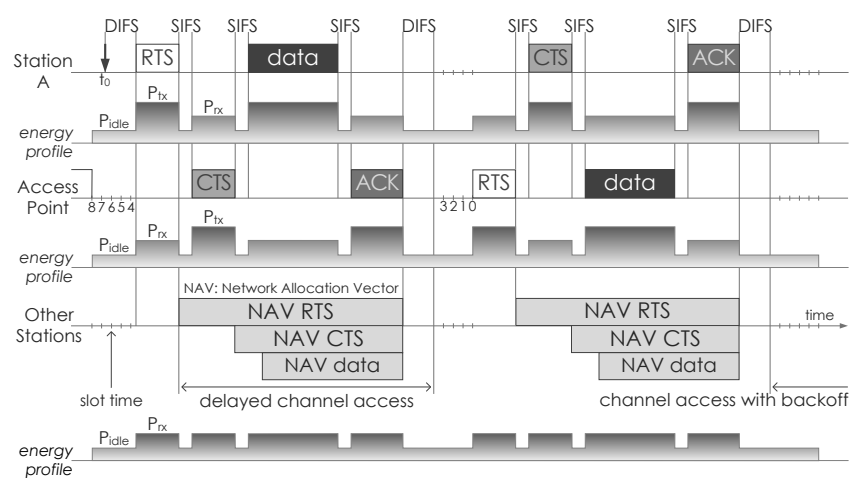

(a) DCF: the RTS/CTS handshake allows a source station to transmit data to an intended destination.

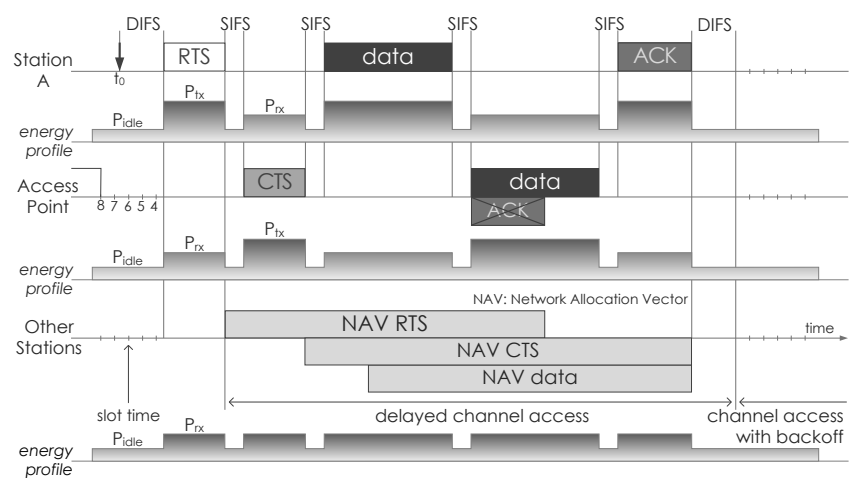

(b) BD-DCF: the RTS/CTS handshake can be used to exchange data between a source station and an intended destination.

Fig. 2. An example of operation and energy consumption of the BD-DCF and DCF protocols in an infrastructure-based WLAN, where Station A and the AP exchange data by using the RTS/CTS access method.

packets. After a SIFS, Station A sends an ACK back to the AP to conclude the data exchange.

To fully leverage the advantages of bidirectional transmissions, we also specify that the AP can send a data packet to another mobile station in case of having no data to exchange with the source station. In this scenario, the basic operation of the BD-DCF remains the same except for when the AP sends the data packet to another destination, which immediately replies with an ACK after successful reception. Bidirectional transmissions can be opportunistically exploited by both the AP and the mobile stations. Since the AP has to compete for channel access with all the mobile stations, it is likely that a mobile station will obtain the access earlier. The uplink transmission can then be used by the AP to have a granted channel access without performing channel contention. Since the AP carries data traffic for all the mobile stations, the fairness in the downlink can be significantly improved.

During the whole data exchange (see Fig. 2b), the other mobile stations have to consume significant energy to listen to the wireless channel until NAV expires before initiating a new channel contention. Since the bidirectional transmission has a longer duration than a single packet transmission, the mobile stations may be able to perform duty-cycling and conserve energy during the time that the wireless channel will be occupied. This strategy is being currently investigated to improve the energy efficiency of mobile stations. 


\section{PERFormance EVAluation}

An open-source computer-based simulator has been implemented in Python to simulate the MAC rules of the protocols under evaluation, i.e. both the DCF and the BD-DCF.

\section{A. Simulation Setup}

The simulation scenario consists of an AP and 20 associated mobile stations. All the mobile stations are located within the transmission range of each other and can only communicate with the AP. Both the AP and the mobile stations generate data packets of constant length according to a Poisson-distributed arrival process. In the downlink, the destination of each data packet is randomly selected among all the mobile stations, all of them with equal probability of being selected. All the packets are assumed to be received with no errors. To balance the uplink and the downlink, the AP is assumed to carry the amount of traffic corresponding to the total traffic load of all the mobile stations. For example, if each mobile station generates $200 \mathrm{kbps}$, the aggregated traffic generated by the 20 mobile stations will be $4 \mathrm{Mbps}$. Therefore, the traffic load of the AP will also be $4 \mathrm{Mbps}$.

The simulation parameters shown in Table I correspond to one of the possible configurations of the IEEE 802.11g [3]. The Extended Inter Frame Space (EIFS) is the time interval which follows after packet collisions. The transmission times of control packets are obtained for the lowest basic rate of 6 Mbps. Both the control rate for the MAC header and the data rate for the payload are used to calculate the transmission time of the MAC Protocol Data Unit (MPDU). The values of power consumption are taken from [13]. All simulation runs were repeated 10 times for the duration of $15 \mathrm{~s}$.

\section{B. Simulation Results}

The results presented in this section show the energy efficiency expressed in transmitted useful bits per consumed Joule considering different values for the traffic load, traffic symmetry, MPDU size, and PHY data transmission rate.

Fig. 3a shows the network energy efficiency as a function of the total offered traffic load. When the channel activity is low, the stations transmit normally and consume little energy since they are idle most of time. As the traffic load increases, the wireless channel becomes more congested and packet collisions occur more frequently. The stations have to wait longer to have a transmission opportunity and consume significant energy to monitor channel activity. In the BD-DCF network, the overall contention in the network is reduced, since a station is allowed to transmit whenever it has received a data packet. This directly translates into higher energy efficiency as the stations consume less energy for channel contention prior to transmitting data. An improvement of up to $29 \%$ can be achieved in saturation conditions.

The energy efficiency of the downlink is shown in Fig. 3b. In the DCF network, channel access opportunities are equally distributed among all the mobile stations and the AP. When the channel contention is low, the AP can deliver all the data to the mobile stations. As the overall contention increases, the AP
TABLE I

SIMULATION PARAMETERS.

\begin{tabular}{c|c|c|c}
\hline Parameter & Value & Parameter & Value \\
\hline SIFS & $10 \mu \mathrm{s}$ & Service & 6 bits \\
DIFS & $28 \mu \mathrm{s}$ & Tail & 16 bits \\
EIFS & $86.33 \mu \mathrm{s}$ & RTS & 20 bytes \\
Slot Time & $9 \mu \mathrm{s}$ & CTS, ACK & 14 bytes \\
Preamble & $16 \mu \mathrm{s}$ & MAC Header & 34 bytes \\
Signal & $4 \mu \mathrm{s}$ & MPDU & 1500 bytes \\
Signal Extension & $6 \mu \mathrm{s}$ & Data Rate & 54 Mbps \\
Time of RTS & $56.33 \mu \mathrm{s}$ & Control Rate & 6 Mbps \\
Time of CTS, ACK & $48.33 \mu \mathrm{s}$ & Transmit Mode & 1.65 Watts \\
Time of MPDU & $292.19 \mu \mathrm{s}$ & Receive Mode & 1.4 Watts \\
$C W_{\min }, C W_{\max }$ & 16,1024 & Idle Mode & 1.15 Watts \\
\hline
\end{tabular}

requires more time and energy to obtain channel access since it has to compete with all the mobile stations. As a result, energy efficiency is reduced significantly. In the BD-DCF network, the AP can have a fair channel access as the mobile stations transmit data more frequently. Energy efficiency can be significantly increased up to a gain factor of 13 .

The average energy efficiency of the uplink is shown in Fig. 3c. As the traffic load increases, mobile stations are more likely to capture the wireless channel in the DCF network. This leads to higher energy efficiency for the mobile stations since they have to spend less energy to sense channel activity before obtaining a new channel access. In the BD-DCF network, channel access opportunities are fairly distributed among the AP and the mobile stations. Since the AP has data to deliver to all the mobile stations, it occupies the wireless channel for a significant longer time. Therefore, mobile stations have to listen to the wireless channel longer before transmitting. As a result, energy efficiency is reduced by $32 \%$ to balance the uplink and the downlink. These results argue the need to develop a strategy combined with bidirectional communications to improve the energy efficiency of mobile stations.

Fig. 3d shows the maximum network energy efficiency as a function of the symmetry coefficient $(\alpha)$ between the uplink $\left(\lambda_{U L}\right)$ and the downlink $\left(\lambda_{U L}\right)$ aggregated traffic. If $\alpha=0$ then $\lambda_{D L}=\lambda_{U L} / n$, where $n$ is the number of mobile stations. Otherwise, $\lambda_{D L}=\alpha \times \lambda_{U L}$. The energy efficiency of the DCF network is not affected by the symmetry coefficient, since all the stations have equal channel access opportunities. For $\alpha=$ 0 , few opportunities for bidirectional communications emerge in the BD-DCF network and the stations have to obtain channel access using the basic DCF most of time. As a result, energy efficiency can be increased slightly by $2 \%$. As $\alpha$ increases, the AP has more data to transmit and both the AP and the mobile stations can benefit from bidirectional communications more frequently to obtain a contention-free channel access. Energy efficiency can be increased up to $30 \%$.

Fig. 3e illustrates the maximum network energy efficiency as a function of the MPDU size. The BD-DCF network always achieves higher energy efficiency than the DCF network. However, the improvement is reduced from $50 \%$ to $25 \%$ as the MPDU size increases. In the BD-DCF network, the AP captures the wireless channel more frequently and the channel access delay experienced by the mobile stations is 


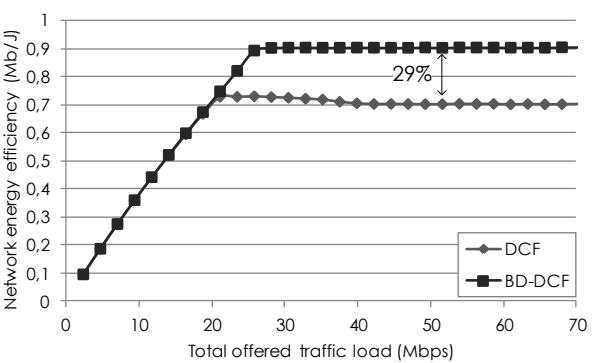

(a) Network energy efficiency.

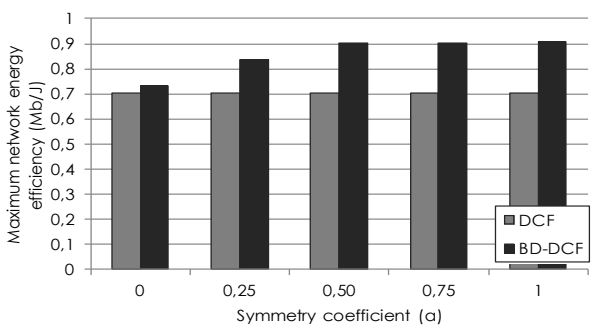

(d) Symmetry coefficient $(\alpha)$.

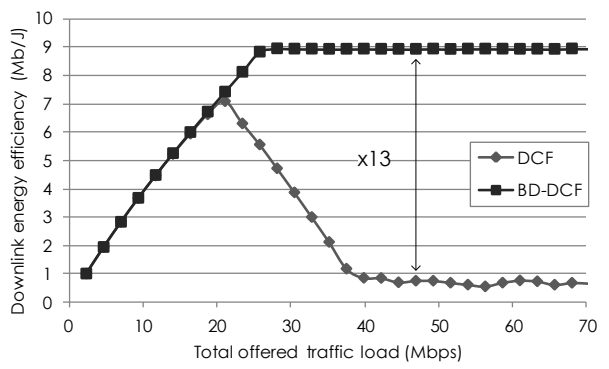

(b) Downlink energy efficiency.

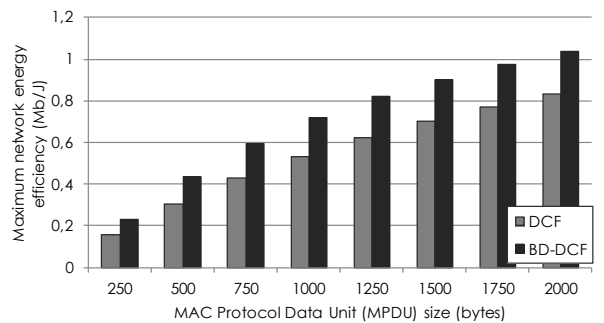

(e) MPDU size.

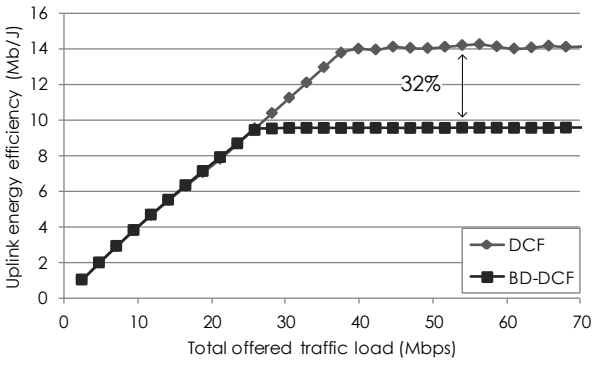

(c) Uplink energy efficiency.

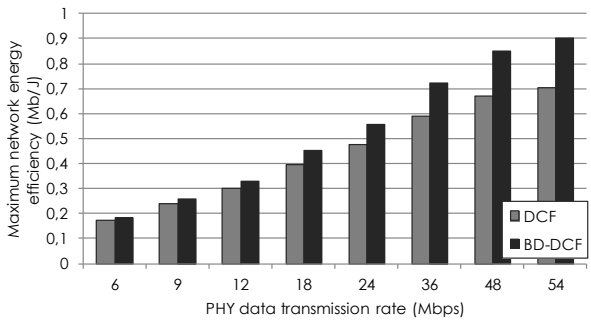

(f) PHY data transmission rate.

Fig. 3. Energy efficiency of the BD-DCF protocol and comparison with the DCF as a function of the traffic load, the symmetry coefficient $(\alpha=100 \%)$, the MPDU size (1500 bytes), and the PHY data transmission rate (54 Mbps).

increased in comparison with the DCF network scenario. This additional delay depends on the duration of the bidirectional transmission, which is proportional to the packet size. When the packet size is small, the mobile stations have to spend less time and energy before transmitting.

The maximum network energy efficiency as a function of the PHY data transmission rate is plotted in Fig. 3f. The BDDCF network achieves higher energy efficiency than the DCF network for all different PHY data transmission rates. For low PHY data transmission rates, the transmission time of a station is increased. This makes the AP occupy the wireless medium for a longer time in the BD-DCF network and the mobile stations have to consume more energy for channel contention. As the PHY data transmission rate increases, the time and energy spent to obtain channel access is reduced. The maximum gain varies between $6 \%$ and $30 \%$.

\section{CONCLUSIONS}

A comprehensive performance evaluation of the BD-DCF protocol has been presented in this paper. This protocol improves the energy efficiency of infrastructure-based WLANs based on DCF by better balancing the channel access opportunities between the AP in the downlink and the mobile stations in the uplink. Computer-based simulations show that BD-DCF outperforms the legacy DCF. The energy efficiency of data transmissions is improved in all cases and can reach up to $50 \%$ of gain with respect to DCF. The specific gain in each case depends on: (i) the degree of traffic symmetry between the uplink and the downlink; (ii) the size of data packets; and (iii) the PHY data transmission rate.

Motivated by the promising results presented in this paper, ongoing work is aimed at exploiting longer transmission times, due to bidirectional links, to let mobile stations not involved in the exchange of data to duty-cycle. This will considerably reduce the energy consumption of mobile stations and will lead to longer life times of batteries.

\section{REFERENCES}

[1] K. M. Gomez, R. Riggio, T. Rasheed, D. Miorandi, and F. Granelli, "Energino: A Hardware and Software Solution for Energy Consumption Monitoring," in IEEE WiOpt, May 2012, pp. 311-317.

[2] G. Perrucci, F. Fitzek, and J. Widmer, "Survey on Energy Consumption Entities on the Smartphone Platform," in IEEE VTC, May 2011, pp. $1-6$.

[3] IEEE, Part 11: Wireless LAN Medium Access Control (MAC) and Physical Layer (PHY) Specifications, IEEE 802.11 Std., 2007.

[4] S.-L. Tsao and C.-H. Huang, "Review: A Survey of Energy Efficient MAC Protocols for IEEE 802.11 WLAN," ACM Computer Communications, vol. 34, no. 1, pp. 54-67, Jan. 2011.

[5] H. Wu, Y. Peng, K. Long, S. Cheng, and J. Ma, "Performance of Reliable Transport Protocol over IEEE 802.11 Wireless LAN: Analysis and Enhancement," in IEEE INFOCOM, vol. 2, Jun. 2002, pp. 599-607.

[6] D.-H. Kwon, W.-J. Kim, and Y.-J. Suh, "A Bidirectional Data Transfer Protocol for Capacity and Throughput Enhancements in Multi-rate Wireless LANs," in IEEE VTC, vol. 4, Sep. 2004, pp. 3055-3059.

[7] W. Choi, J. Han, B. J. Park, and J. Hong, "BCTMA (Bi-directional CutThrough Medium Access) Protocol for 802.11-based Multi-hop Wireless Networks," in ACM ISSADS, Jan. 2005, pp. 377-387.

[8] M. Ozdemir, G. Daqing, A. B. McDonald, and J. Zhang, "Enhancing MAC Performance with a Reverse Direction Protocol for High-Capacity Wireless LANs," in IEEE VTC, Sep. 2006, pp. 1-5.

[9] C. Crespo, J.Alonso-Zarate, L. Alonso, and C. Verikoukis, "Distributed Point Coordination Function for Wireless Ad hoc Networks," in IEEE VTC, Apr. 2009, pp. 1-5.

[10] J. Alonso-Zarate, C. Crespo, C. Skianis, L. Alonso, and C. Verikoukis, "Distributed Point Coordination Function for IEEE 802.11 Wireless Ad Hoc Networks," Ad Hoc Networks, vol. 10, no. 3, pp. 536-551, 2012.

[11] N. S. P. Nandiraju, H. Gossain, D. Cavalcanti, K. R. Chowdhury, and D. P. Agrawal, "Achieving Fairness in Wireless LANs by Enhanced IEEE 802.11 DCF," in IEEE WiMob, Jun. 2006, pp. 132-139.

[12] R. Palacios, F. Granelli, D. Gajic, and A. Foglar, "An Energy-Efficient MAC Protocol for Infrastructure WLAN Based on Modified PCF/DCF Access Schemes Using a Bidirectional Data Packet Exchange," in IEEE CAMAD, Sep. 2012, pp. 216-220.

[13] E.-S. Jung and N. H. Vaidya, "An Energy Efficient MAC Protocol for Wireless LANs," in IEEE INFOCOM, vol. 3, Jun. 2002, pp. 1756-1764. 